\title{
Transoral Robotic Surgery
}

National Cancer Institute

\section{Source}

National Cancer Institute. Transoral Robotic Surgery. NCI Thesaurus. Code C94439.

A minimally invasive procedure in which a surgeon performs surgical neck procedures

through the mouth using a robotic surgical system. 